\title{
Retroreflecting Optical Modulator Using an MEMS Deformable Micromirror Array
}

\author{
Trevor K. Chan and Joseph E. Ford
}

\begin{abstract}
A modulating corner-cube reflector with one microelectromechanical-system (MEMS) mirror that deforms from a flat into a hexagonal array of concave reflective microlenses to disperse the retroreflected wavefront is demonstrated. It is shown that such retromodulators can operate under a wide range of wavelength and angle illumination using Huygens-Fresnel propagation analysis, and this analysis is verified using devices fabricated by surface micromachining. A gold-coated silicon-nitride membrane suspended over 1-mm-diameter circular cavities had a resonant frequency of $160 \mathrm{kHz}$ and $0.55-\mu \mathrm{m}$ maximum deformation with $79 \mathrm{~V}$ applied. While this deflection was only $2 / 3$ of the design value of $0.8 \mu \mathrm{m}$, we measured an up to 7:1 modulation contrast ratio from a prototype retromodulator, which achieved $100-k H z$ modulation over a 100-nm optical bandwidth, a $35^{\circ}$ range of incident angles, and temperatures ranging from 20 to $100{ }^{\circ} \mathrm{C}$.
\end{abstract}

Index Terms-Microelectromechanical devices, modulation, optical communication, optical diffraction, remote sensing.

\section{INTRODUCTION}

$\mathbf{F}$ REE-SPACE optical (FSO) communications combines aspects of both wireless communication and high-bandwidth fiber communication by directing an optical beam between two line-of-sight points to establish a bidirectional communication link. Placing an optical retroreflector at the remote end causes a self-aligned portion of the outgoing beam to return to the source, subject only to diffraction and scattering losses. A retromodulator is a retroreflector with an electrically controlled signal intensity at the original source point. Retromodulators can operate by absorbing the incident signal, or by scattering the reflected wavefront so that it does not propagate back to the source. A retromodulator can effectively reuse the incident optical power and alignment to encode a data signal on the return beam, drastically simplifying the remote opticalcommunications terminal. Fig. 1 shows the overall system architecture.

Our target application is remote telemetry, which includes remotely interrogated environmental sensors and secure-fieldcommunications links. Environmental sensors may be distributed over a large geographic area, then remotely interrogated by a laser scanning from an aircraft. Field-communications links can be used for military identification ("identify friend or foe") and secure data transmission. In both applications,

Manuscript received January 21, 2005; revised August 1, 2005. This work was supported by the Cubic Corporation and the Natural Sciences and Engineering Research Council of Canada.

The authors are with the Department of Electrical and Computer Engineering, University of California, San Diego, CA, 92093-0407 USA (e-mail: tkc@ucsd.edu; jeford@ucsd.edu).

Digital Object Identifier 10.1109/JLT.2005.859853 optical retromodulators offer potential advantages over conventional radio links in terms of power dissipation and selectivity.

Several retromodulator characteristics directly impact overall communications-system performance: wavefront aberration and effective optical aperture affect maximum operational range; modulation response time and contrast ratio determine data bandwidth; and operational tolerances including modulator acceptance angle, wavelength range, and ability to withstand physical and thermal shock determine overall system utility and robustness. In remote telemetry, the communications range may extend from 0.1 to $10 \mathrm{~km}$, and must operate under varying environmental conditions. This means that the effective optical aperture must be $1 \mathrm{~cm}$ or larger, and the wavefront distortion should be below $\lambda / 10$. Further, we require that the system operate under a wide range of incident angles: up to $\pm 30^{\circ}$ is possible with a hollow corner-cube retroreflector. To allow spread-spectrum and wavelength-agile systems, and to avoid excessive system costs, we want the modulator to function over the 1.45 to $1.55-\mu \mathrm{m}$ spectrum in the eye-safe communications range. Finally, we prefer a data-modulation contrast of 10:1 (2:1 required) and a data-modulation rate of at least $100 \mathrm{kHz}$. Data rates as high as gigahertz are desirable, but a wide range of remote-telemetry-application requirements can be satisfied with $100-\mathrm{kHz}$ modulation.

Previous microelectromechanical-system (MEMS) retromodulators demonstrated that using a full-aperture tilting mirror [1] successfully yielded large contrast ratios. Their functional angular range is limited only by the acceptance angle of the corner cube. However, the electromechanical switching of the tilting mirror requires a switching time that is a function of the mirror size. This approach limits the response time of larger aperture (long range) retromodulators to milliseconds. Another successful MEMS retromodulator used a diffractive structure [2], a reflective diffractive grating that is electromechanically switched into a flat refractive surface. This grating light modulator provides faster modulation $(\sim 1 \mathrm{MHz})$, since very little mechanical deflection $(\sim \lambda / 4$ or $0.3 \mu \mathrm{m})$ is required. However, this modulator requires wavelength and angle matching, which limited the operating-angle range to $6^{\circ}$ even for low $(0.4 \mathrm{~dB})$ contrast. So far, we have not achieved the desired combination of speed, aperture, and wavelength and angle insensitivity.

Reflective MEMS etalons using a drumhead structure with a variable air gap have been extensively investigated for use in telecommunications as data modulators [3] and broad-spectrum variable attenuators [4]. The response time of such devices depends on several parameters, including the drum diameter and membrane tension, but have been demonstrated to operate 


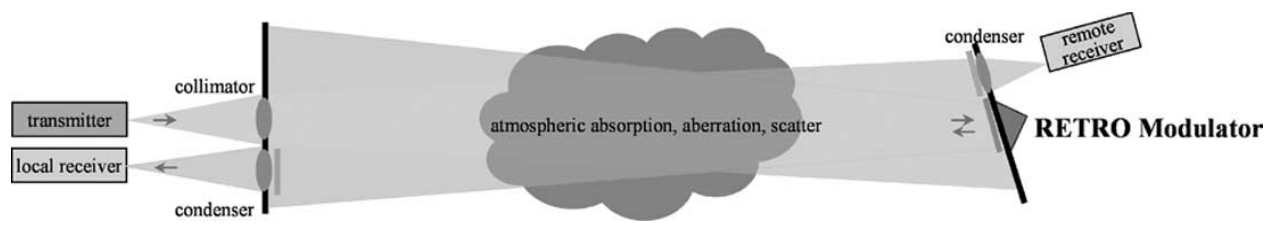

Fig. 1. Bidirectional communications system using automatic retroalignment.

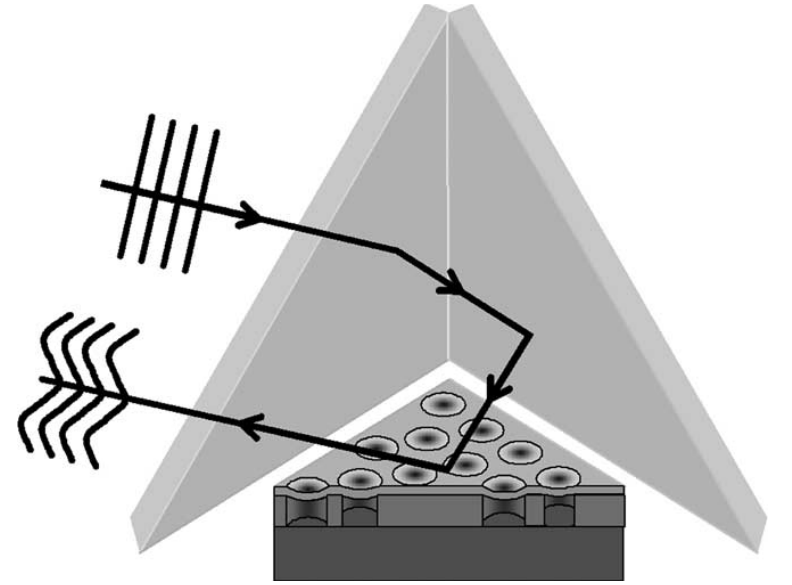

Fig. 2. Diagram of the MEMS deformable mirror, showing a section of the device to reveal air pockets that allow deformation at selected areas.

at well over $1 \mathrm{MHz}$ [5]. This is still orders of magnitude slower than multiple-quantum-well modulators [6], but the MEMS devices are also far less expensive to manufacture (especially for large apertures) and less sensitive to operating wavelength and temperature. In fact, a large-aperture MEMS etalon modulator based on this design and intended for use with cat's-eye retroreflector optics was recently successfully tested [7].

In this paper, we present theoretical and experimental results on an MEMS device based on the same device structure but modified to act as a first-surface deformable mirror rather than an etalon. When this device is assembled into a corner cube, the result is a highly efficient wavelength- and angle-insensitive retromodulator.

\section{Deformable-Micromirror-Array CONCEPT AND DESIGN}

The basic device, shown in Fig. 2, is a first-surface MEMS deformable mirror switching from a flat to a hexagonal array of concave microlenses. This device concept was first proposed in 1996 [8], and a somewhat different rectangular structure was tested in [9]. When the device is structured as an array of spherical microlenses, the modulator operates midway between the geometrical and diffractive regimes; light distribution is substantially determined by geometrical curvatures, but interference between multiple diverging beams produces strong diffraction effects that suppress the zero-order reflectivity. The microlenses are separated by $1-\mathrm{mm}$ pitch and have a $75 \%$ deformable surface area. The active area, when arrayed in smaller deformable sections, achieves greater lenslet curvatures and shorter switching times. This allows for the scaling of the device to large $(10 \mathrm{~cm}+)$ apertures without sacrificing speed or diffraction efficiency.
We calculated the diffractive behavior of the lenslet-arrayed micromirror using Huygens-Fresnel diffraction theory on a single-deformable-mirror structure. The other two mirrors of the corner cube were not included in these calculations for simplicity in understanding the deformable mirror's behavior. The device surface profile was modeled and sampled by a $250 \times$ 250 grid and the resulting phased signals propagated $6.6 \mathrm{~m}$ to the readout plane, effectively in the far field. Fig. 3(a) and (b) shows the resulting far-field diffraction patterns from the flat and deformed mirror when it is illuminated by a plane 1550-nm-wavelength wavefront at normal incidence. The zeroth-order diffracted beam is shown at the center of the figures. Both figures use a logarithmic scale to best display the diffracted far-field pattern. We see a hexagonal array of spots in an envelope created by the refractive aperture of the individual microlenses. The far-field diffraction pattern is the Fourier transform of the deformable-mirror surface. Each spot in the array replicates the original reflected beam shaped by the overall mirror-array aperture. The spots are separated by a pitch inversely proportional to the array spacing of the deformable mirror lenslets.

Fresnel diffraction theory predicts the near-linear relationship (1) between the period of the arrayed diffraction pattern and the spatial frequency $(1 / \mathrm{T})$ of the microlens array. Fig. 3(c) and (d) show the line profile of the far-field diffraction pattern where Fig. 3(d) is calculated with 1500- and 1600-nm light. These two line profiles are difficult to distinguish; showing that the simulations predict low wavelength sensitivity. Unlike a single-wave-delay diffractive MEMS modulator, the diffraction pattern scales with wavelength by $4.0 \mu \mathrm{rad} / \mathrm{nm}$, while maintaining approximately the same contrast

$$
\tan \theta_{\text {period }}=\frac{\lambda}{T \cos \left(\frac{\pi}{6}\right)}
$$

For FSO communications, the signal energy collected by the original source transmitter is proportional to the power in the zeroth-order diffracted beam. In all of our simulations, the detector aperture is taken around this beam. Fig. 4 shows the computed relative power incident on the detector as we increase the device deformation. This is shown for different mirror tilt angles $\alpha$, which are also the angles of incident light. An increase in the deformation immediately results in a decrease of power at the detector for all incident angles. With further deformation, the detected power oscillates, typical of diffraction phenomena, and dampens to a lower intensity. The detected power does not approach zero since parts of the deformable mirror remain flat and always reflect light into the detector. We can note that a $10-\mathrm{dB}$ contrast ratio is 


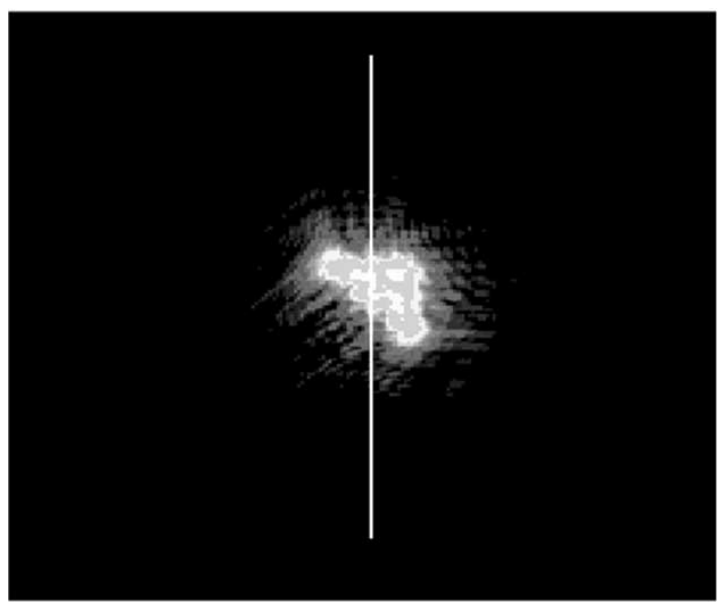

(a)

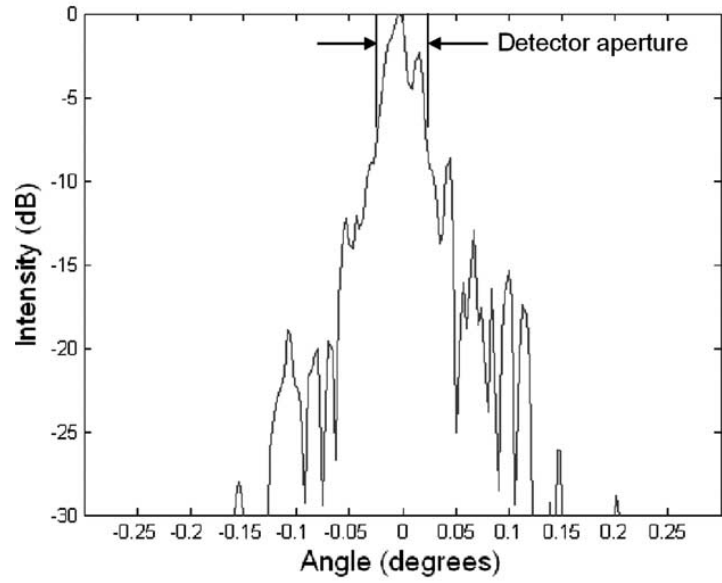

(c)

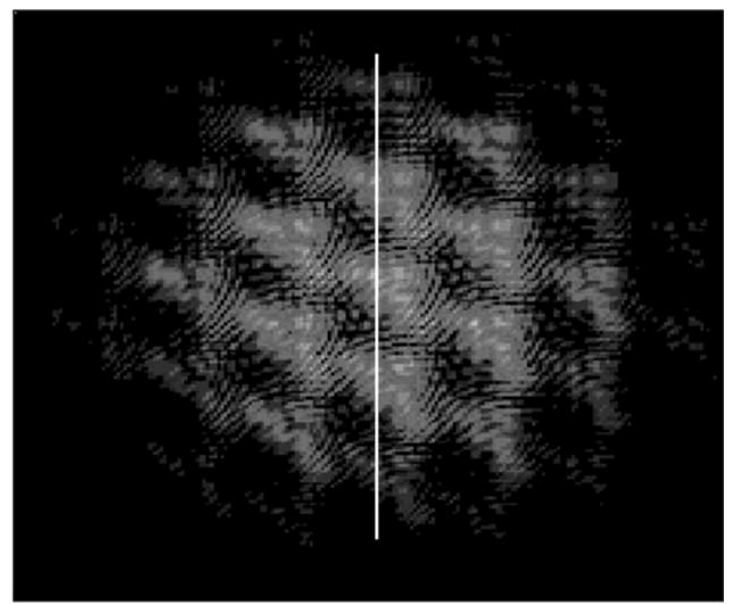

(b)

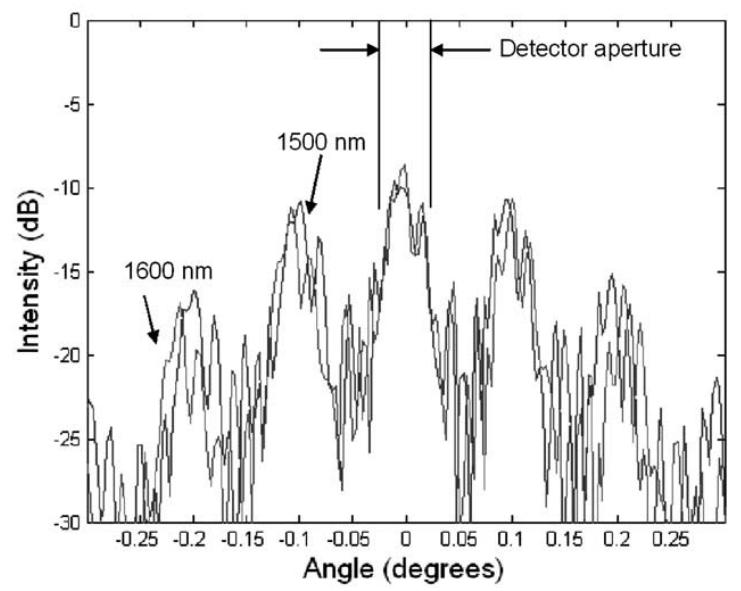

(d)

Fig. 3. Calculated far-field patterns (normalized) with (a) 0 deflection and (b) $0.55-\mu \mathrm{m}$ deflection and their line profiles (c) and (d). The line profile from the deformed mirror (d) is calculated using 1500- and 1600-nm light to demonstrate the wavelength insensitivity of diffraction. Photos and plots both use a logarithmic intensity scale.

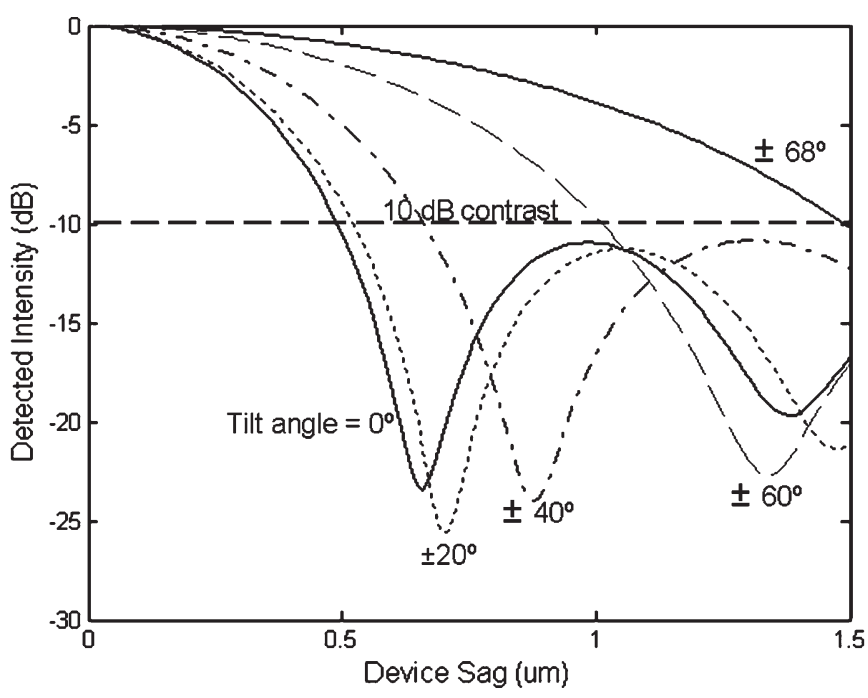

Fig. 4. Relative intensity reaching the detector as a function of the mirror deformation. This relationship is shown at various mirror-tilt angles.

maintained for incident angles up to $\pm 68^{\circ}$ at $1.5-\mu \mathrm{m}$ deformations. This demonstrates that the deformable mirror will work as a modulator for a large range of angles. The three mirrors in a corner-cube retroreflector are each oriented $54.7^{\circ}$ to the normal of the corner cube.

A single deformable MEMS mirror can replace a mirror in the corner cube to form a retroreflecting modulator. Furthermore, all three of the mirrors can be deformable MEMS mirrors. In this case, Fig. 4 predicts a $10-\mathrm{dB}$ contrast with a $0.8-\mu \mathrm{m}$ sag for the entire angular aperture of the corner cube. Further calculations verify that these contrast ratios are maintained for increasing propagation distances of at least $1 \mathrm{~km}$. A mechanical deflection of less than $0.1 \%$ of the membrane width, combined with the noncontact nature of the structure, means that this device will be extremely robust to mechanical shock and sustained high-frequency operation.

\section{EXPERIMENTAL DEMONSTRATION OF THE DEFORMABLE-MICROMIRROR ARRAY}

Our devices were fabricated by MEMSCAP using nowstandard MEMS processes [3]-[6]. The layered structure of the device is illustrated in Fig. 5. A silicon substrate was covered with a $3-\mu \mathrm{m}$ sacrificial layer of phosphosilicate glass (PSG), and a $1-\mu \mathrm{m}$ active structural layer of silicon nitride. The mechanically active "drum" regions were defined by patterning the 


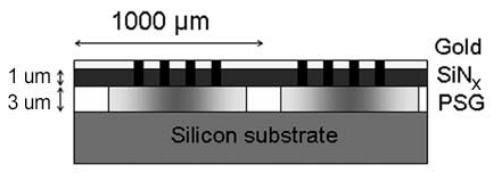

(a)

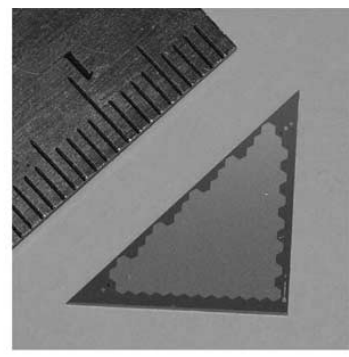

(b)

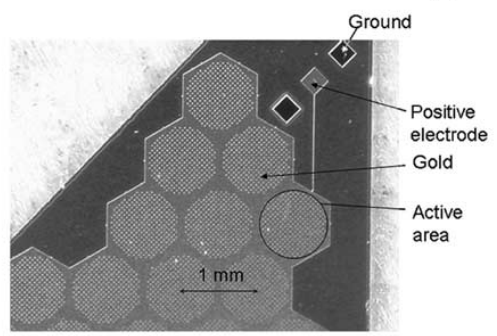

(c)

Fig. 5. (a) Layered device diagram, (b) fabricated die, and (c) close-up of individual micromirror cells.

nitride with sets of etch access holes arranged into a hexagon array on a 1-mm pitch. The etch access holes in the samples tested were $5 \mu \mathrm{m}$ in diameter, distributed in a square array with a $46-\mu \mathrm{m}$ center-to-center pitch. Reactive ion etching was used to cut through the nitride into the sacrificial layer, then a timed isotropic etch undercut the active layer to release the freestanding circular drumheads. The top gold mirror, with roughly $95 \%$ reflectivity in the near-infrared wavelength of interest, was deposited on the released structure.

An applied voltage between the membrane and substrate layers causes an electrostatic attraction that pulls the membrane from its normal flat state, producing a dip in the reflective surface wherever there exists a pocket in the PSG layer. The honeycomb pattern of pockets in the PSG layer creates the hexagonal array of dips in the gold layer. Each dip acts as a concave reflective lens. The resonant frequency of this device was measured to be $160 \mathrm{kHz}$. Increasing the nitride-film stress and drive voltage can increase the device response to above $1 \mathrm{MHz}$.

We first characterized the deformable mirror by substituting it as one of the mirrors in a Michelson's interferometer. Thus, reflected 1550-nm light was combined with a plane wave to give an interference pattern corresponding to the deformations of our mirror. These patterns allowed us to extrapolate the profile of the deformable-mirror surface, shown in Fig. 6. The first experimental samples achieved a maximum sag of $0.55 \mu \mathrm{m}$ with an applied voltage of $79 \mathrm{~V}$. This deformation limit is caused by an error in the deposition composition of the $\operatorname{SiN}_{x}$ layer, making the deformable membrane conductive and somewhat nonuniform in layer stress. This caused the deformation to be nonuniform on the large $(10 \mathrm{~mm})$ scale of the device and caused an electrical short circuit when we applied significantly more than $79 \mathrm{~V}$ to the device. A spare device was sacrificed to show that arcing from the short circuit punctured the membrane surface, rendering it useless as a reflective mirror. Although we could not produce greater than $0.55-\mu \mathrm{m}$ deformations with this

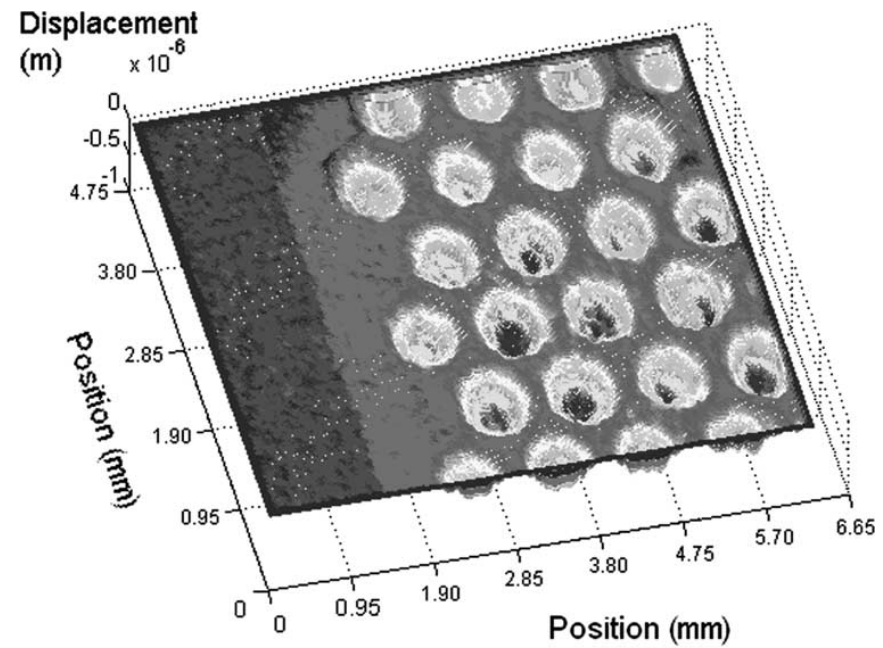

(a)

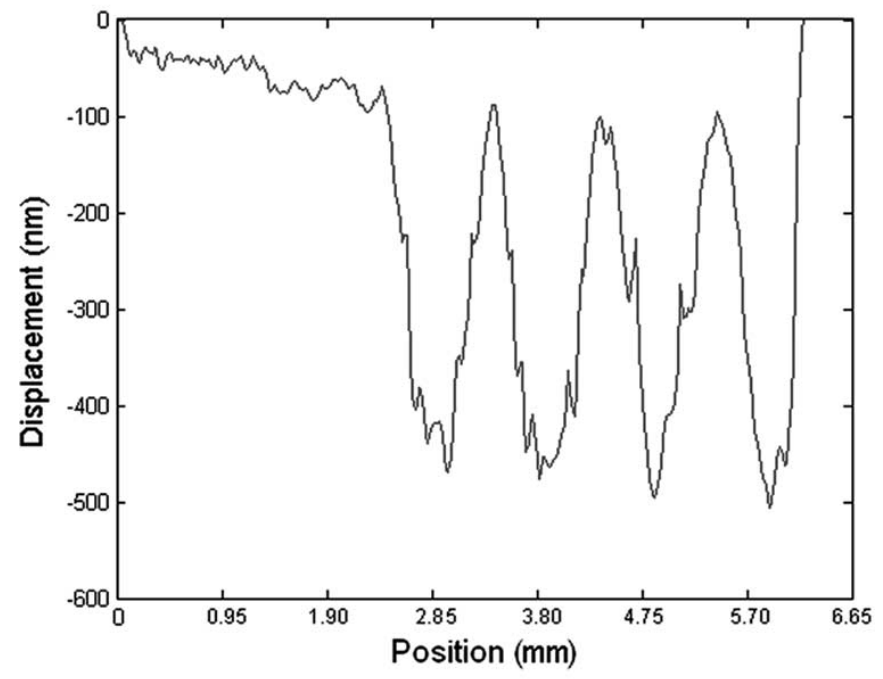

(b)

Fig. 6. (a) Profile of the device surface after deformation, extrapolated from interferometry measurements. (b) Profile of the device deformation.

initial prototype device, all device problems can be corrected upon refabrication with a uniform nonconductive $\operatorname{SiN}_{x}$ layer.

The device was illuminated with a collimated 1460-to1580-nm wavelength-tunable laser and the reflected light was propagated $6.6 \mathrm{~m}$ onto a white screen, effectively in the far field. The intensity pattern was captured on the screen by a Sensors Unlimited 320 M InGaAs camera. Fig. 7(a) and (b) show the logarithmic far-field intensity pattern before and after $0.55-\mu \mathrm{m}$ mirror deformation with near-normal illumination by 1520-nm-wavelength light. These patterns resemble the patterns predicted in the simulations; a repetition of the zerothorder reflected light in a hexagonal array that is confined by a circular envelope. The diffracted spots are noticeably smaller because the mirror was illuminated with an apodized Gaussian beam profile than the aperture of the mirror. Fig. 7(c) and (d) correspond to the intensity-line profiles along the horizontal white lines as indicated. These line profiles reveal the relative intensities of the diffraction patterns. Here, approximately $90 \%$ of the power has been diverted from the original beam into the hexagonal pattern of surrounding beams, yielding 10:1 


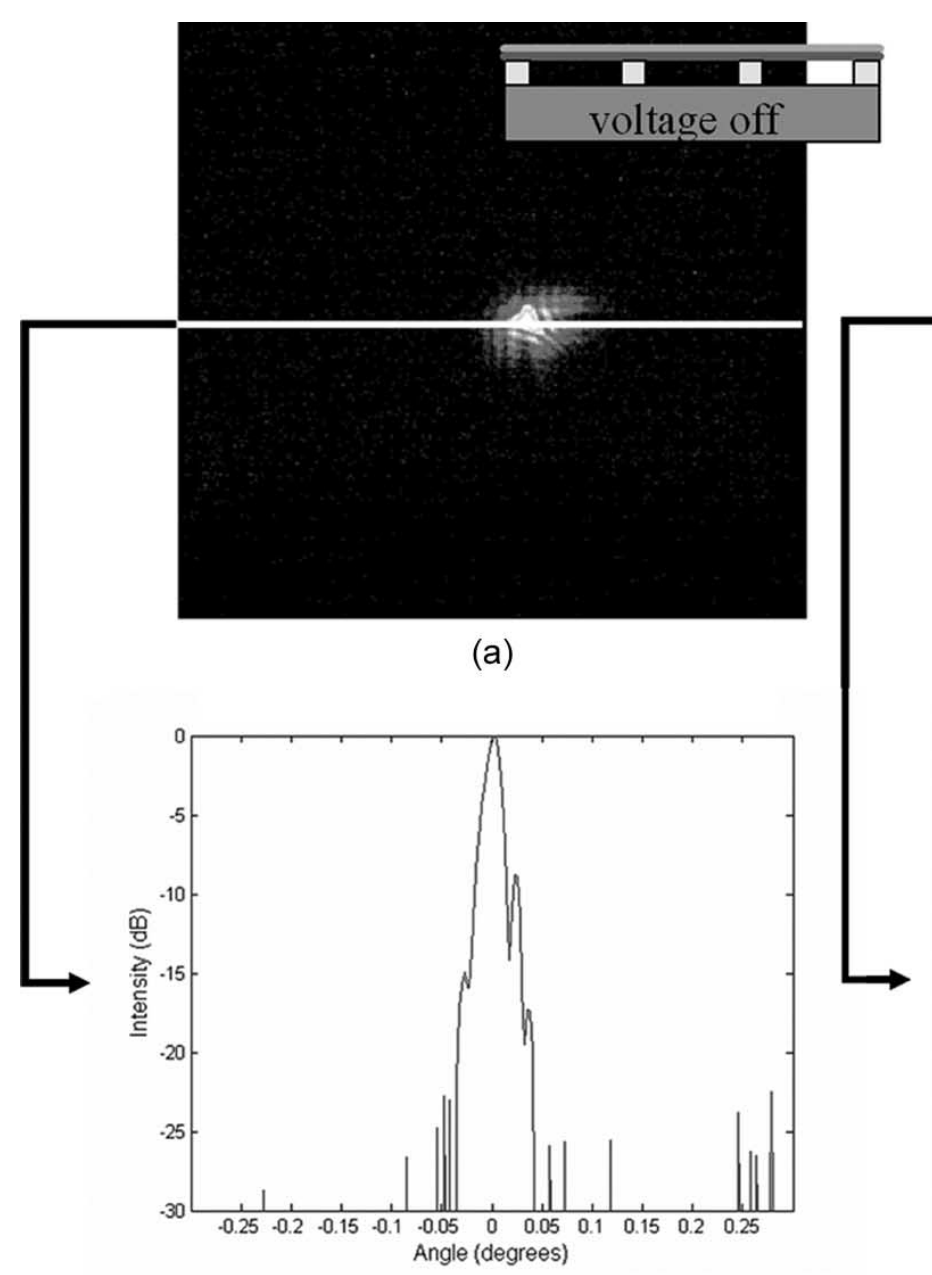

(c)

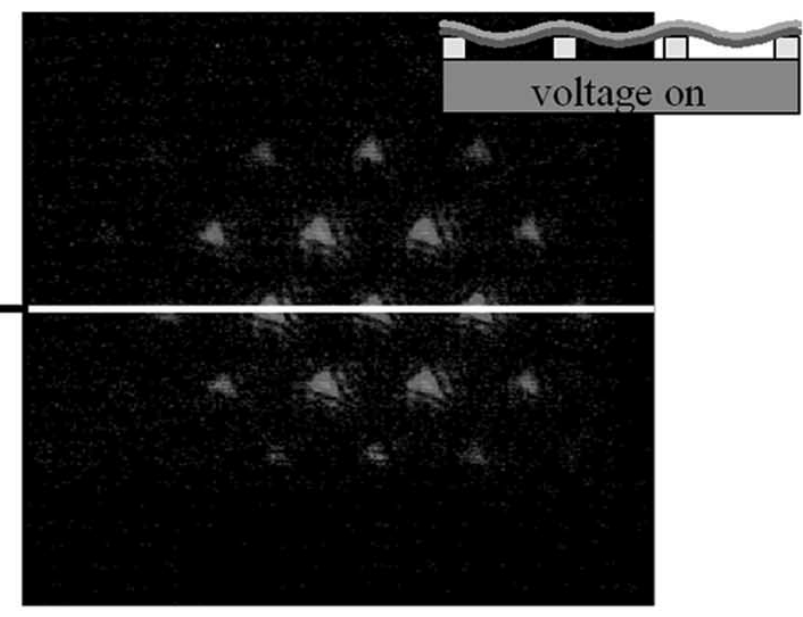

(b)

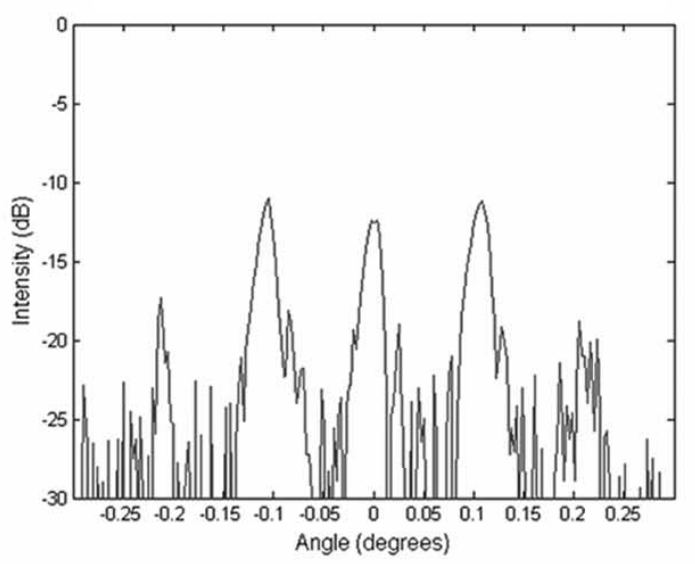

(d)

Fig. 7. Experimentally measured far-field intensity patterns from a triangular-aperture device in the (a) zero-voltage (flat mirror) and (b) off state, and their line profiles (c) and (d). These far-field patterns were observed using a 1520-nm laser light. Photos and plots both use a logarithmic intensity scale.

extinction. These results agree with the theoretical predictions made earlier in this paper. Furthermore, diffraction patterns produced using 1460- and 1575-nm light created far-field diffraction patterns that were indistinguishable from these results, thereby demonstrating wavelength insensitivity.

Fig. 8 shows the measured-line profile for the same far-field diffraction pattern when the deformed mirror was illuminated with a 14-nm-bandwidth amplified-spontaneousemission (ASE) optical source centered at $1532 \mathrm{~nm}$, instead of a monochromatic coherent laser beam. There is a strong correlation between Figs. 7(d) and 8; there are distinct diffraction orders that are separated by approximately $0.11^{\circ}$ divergence and the zeroth-order beam experiences approximately $11-\mathrm{dB}$ extinction. This example further demonstrates the wavelength insensitivity of the deformable mirror; moreover, the deformable mirror functions with temporally incoherent white light.

Experiments verified that our device followed the angular response predicted by our calculations up to its maximum $0.55-\mu \mathrm{m}$ achievable deformation, but a deformation of $0.8 \mu \mathrm{m}$ would be necessary to observe significant diffractive effects at incident angles greater than $40^{\circ}$.

To study the temperature dependence of our devices, a deformable mirror was mounted on a thermoelectric cooler (TEC)

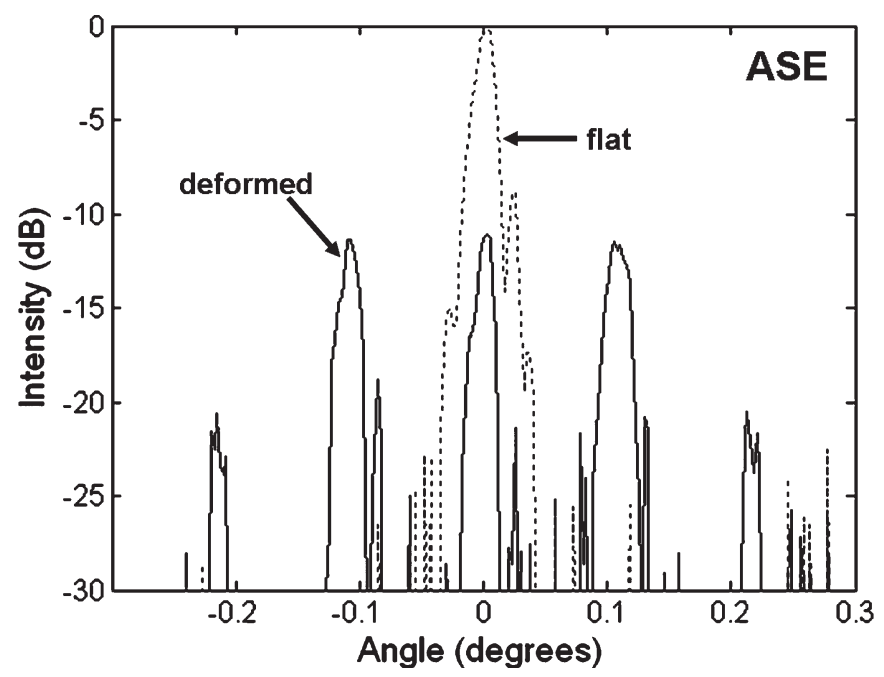

Fig. 8. Line profile of the mirror far-field diffraction pattern using incoherent illumination.

with an attached thermistor. We used a deformable mirror with $50 \%$ (instead of $75 \%$ ) deformable area because these mirror types were readily available for "test to destruction" 


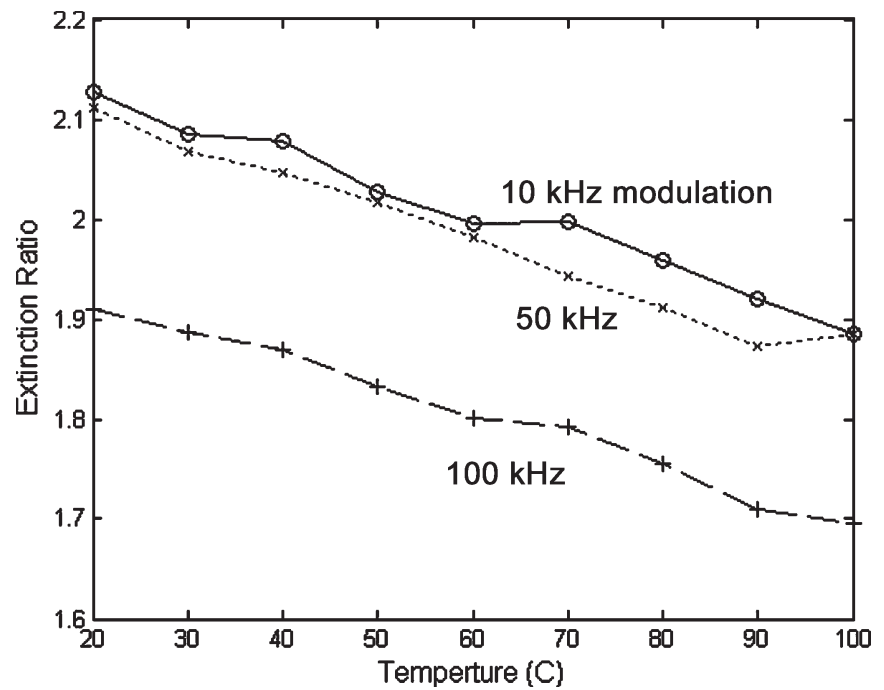

Fig. 9. Optical contrast ratio (extinction) as a function of retromodulator temperature. The modest decline in extinction comes from the thermally induced curvature of the device mounted on the heater.

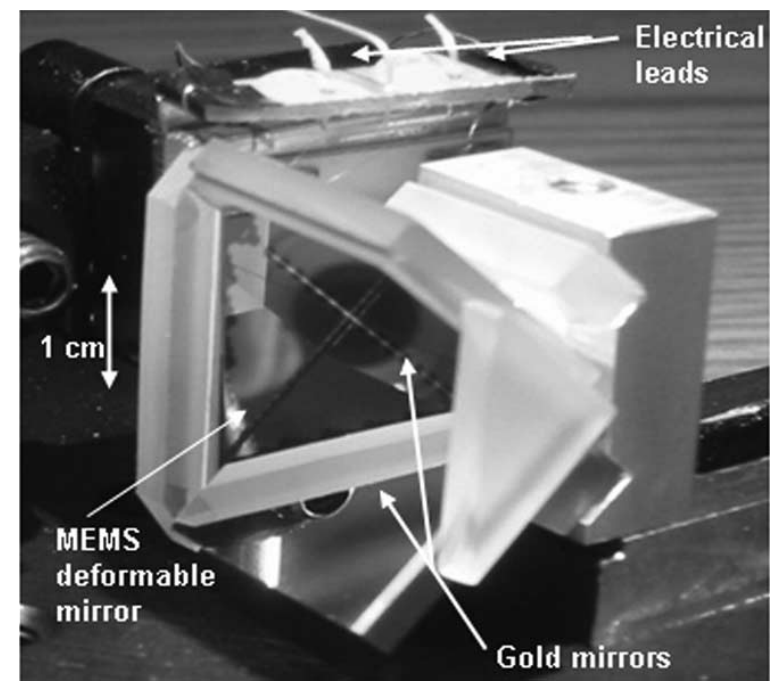

Fig. 10. Photograph of the assembled MEMS deformable-mirror retromodulator.

sacrificing. As you might expect, these 50\%-active-deformablearea devices require more voltage and produce less extinction. The TEC and thermistor allowed for active temperature control during these experiments. The mirror was illuminated with a 1550-nm laser at near-normal incidence and the reflection was propagated $6.6 \mathrm{~m}$ into a collection lens and detector. The detector aperture isolated the zeroth-order diffracted beam and measured its extinction ratio as the device was driven with an 85 -V-amplitude binary signal at 10, 50, and $100 \mathrm{kHz}$. Fig. 9 shows a decrease in the extinction ratios as the device temperature was increased. Further interferometry measurements of the mirror-surface profile revealed that thermal expansion resulted in the bending of the overall surface. The consequential curved-mirror surface reduced signal power in the high-signalpower (flat mirror) state but had less of an effect on the off-signal level when the mirror was sagged. These changes

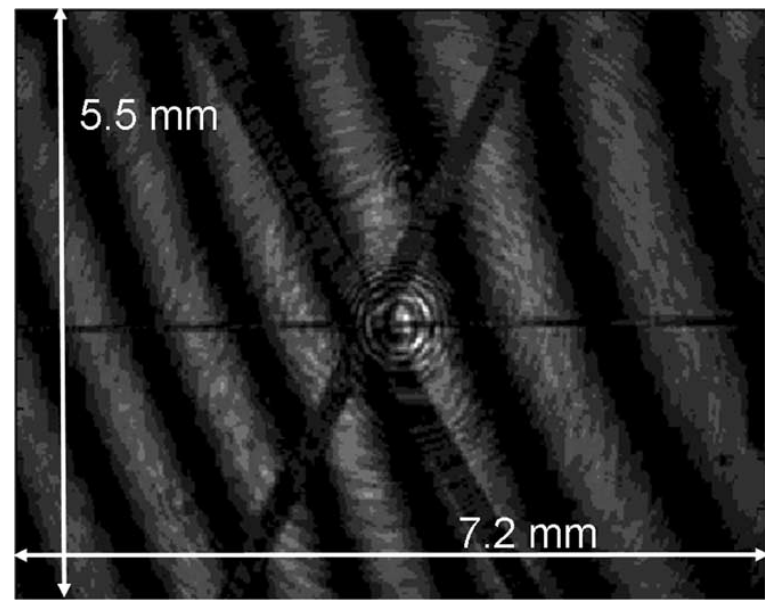

(a)

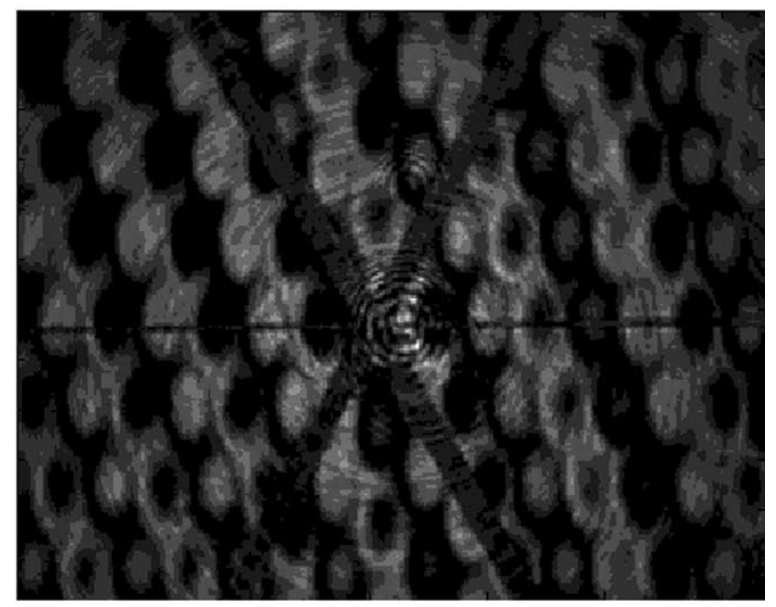

(b)

Fig. 11. Interferometer photographs of the retromodulator surface. (a) Flat ( $\lambda / 9$ aberration). (b) Deformed ( $>\lambda / 2$ aberration).

account for the measured decrease in extinction and can be mitigated with better packaging. Using a similar device, we ramped the temperature to over $400{ }^{\circ} \mathrm{C}$ with no visible impact. These results are compatible with previous measurements on membrane modulators, which were shown to operate in liquid nitrogen and at $400{ }^{\circ} \mathrm{C}$ temperatures.

\section{EXPERIMENTAL DEMONSTRATION OF THE CORNER-CubE RETROMODULATOR}

The deformable mirror was assembled with fixed-dihedral $\left(90^{\circ}\right.$ angle) gold mirrors to form the corner-cube retromodulator shown in Fig. 10. We apply voltage to the deformable mirror through the electrical leads displayed at the top of this photograph. We mounted the deformable mirror on a tilt stage to allow accurate alignment with respect to the other two mirrors.

Light must strike each of the three mirrors for retroreflection. Therefore, there are six different paths for retroreflection; each path corresponds to a different permutation of reflections from three mirrors. Fig. 11(a) is a photograph of the retroreflected light at the retroreflector-mirror surfaces. It is combined with a slightly tilted plane wave that creates the observable 


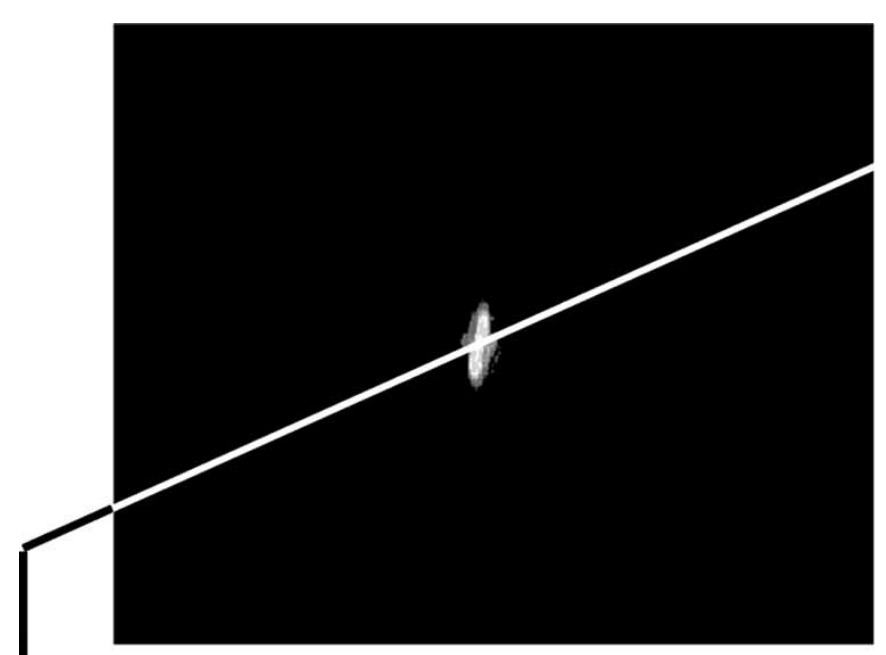

(a)

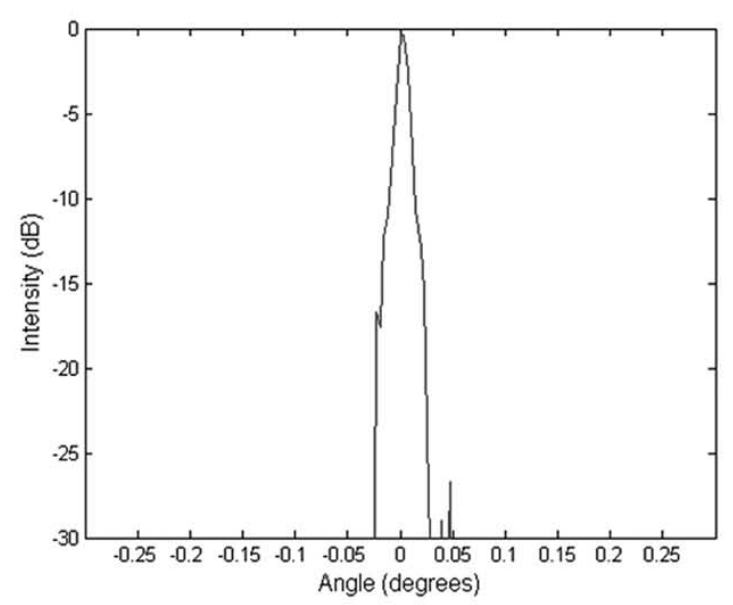

(c)

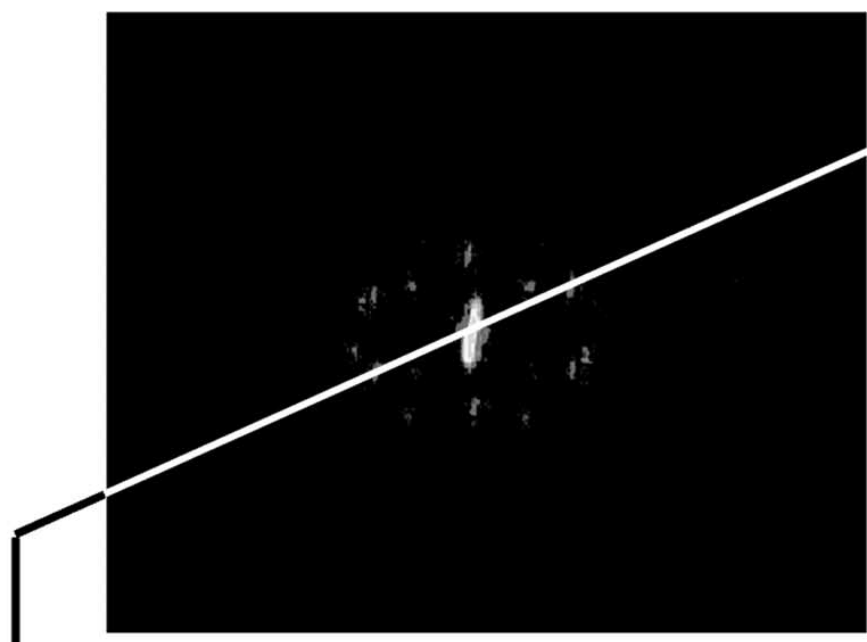

(b)

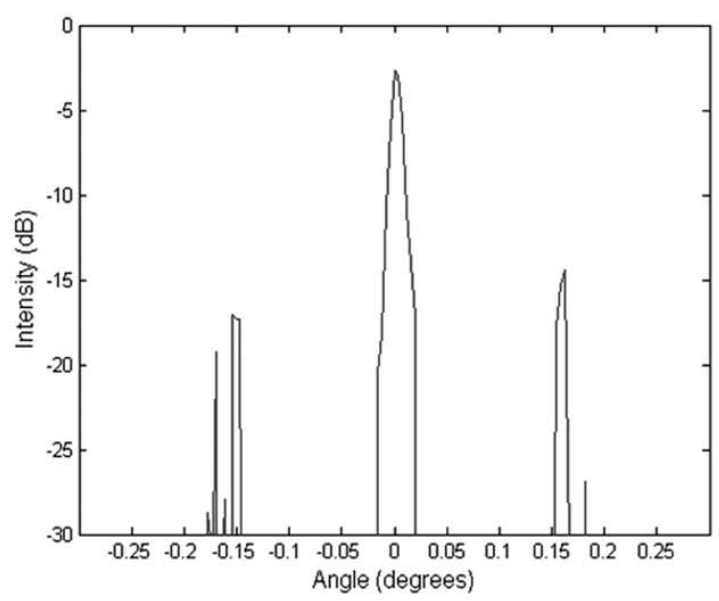

(d)

Fig. 12. Far-field diffraction pattern from the retromodulator with an applied voltage of (a) 0 and (b) 79 V. Photos and plots both use a logarithmic intensity scale.

interference fringes. The reflected light is divided into the six sections by gaps between mirrors of the corner cube; each section corresponds to one of the six different paths of light. Each of these paths reflect off the deformable mirror, adding phase modulation to the entire signal. Fig. 11(b) shows the retroreflection from the deformed device, revealing at least $\lambda / 2$ phase distortion over the entire return beam.

Fig. 12 shows the far-field intensity pattern created by the retromodulator with normal incident light into the corner cube (each mirror normal is oriented at the same angle, $54.7^{\circ}$, from the incident light). This shows several hexagonal arrays that are rotated by different angles and superimposed. Recall that diffraction from only the deformable mirror created a single-hexagonal-array diffraction pattern. The difference comes from the multiple paths of light through the corner cube. Depending on the order of reflections, the phase distortion acquired from the deformable mirror can become inverted at various angles with subsequent reflections within the corner cube. The diffraction pattern is inverted in a way that makes it correspond to the path it takes through the corner cube.
To achieve better contrast, the corner cube is tilted $31.7^{\circ}$ towards its deformable-mirror side such that the incident rays strike the deformable mirror at $23^{\circ}$. Fig. 13 shows the resulting far-field diffraction pattern after this adjustment is made. This orientation is more indicative of minimum performance when using multiple MEMS mirrors instead of a single MEMS mirror with two fixed glass mirrors.

\section{A. Modulation-Time Response}

The retroreflecting modulator was driven by a square signal with 79-V amplitude. In these experiments, the retroreflector was rotated by $31.7^{\circ}$ towards the deformable mirror for high extinction. The retroreflected signal was separated from the incoming beam path using a 50/50 IR beam splitter and collected by a Fourier lens $6.6 \mathrm{~m}$ away. A detector was placed in the Fourier plane of this lens on an axis and only captured the zeroth-order diffracted spot. Fig. 14 shows the detected signal intensity. This shows a 7.1:1 signal contrast with riseand-fall times of 4.3 and $6.8 \mu \mathrm{s}$, respectively. Recall that the deformation was limited by the maximum deformation 


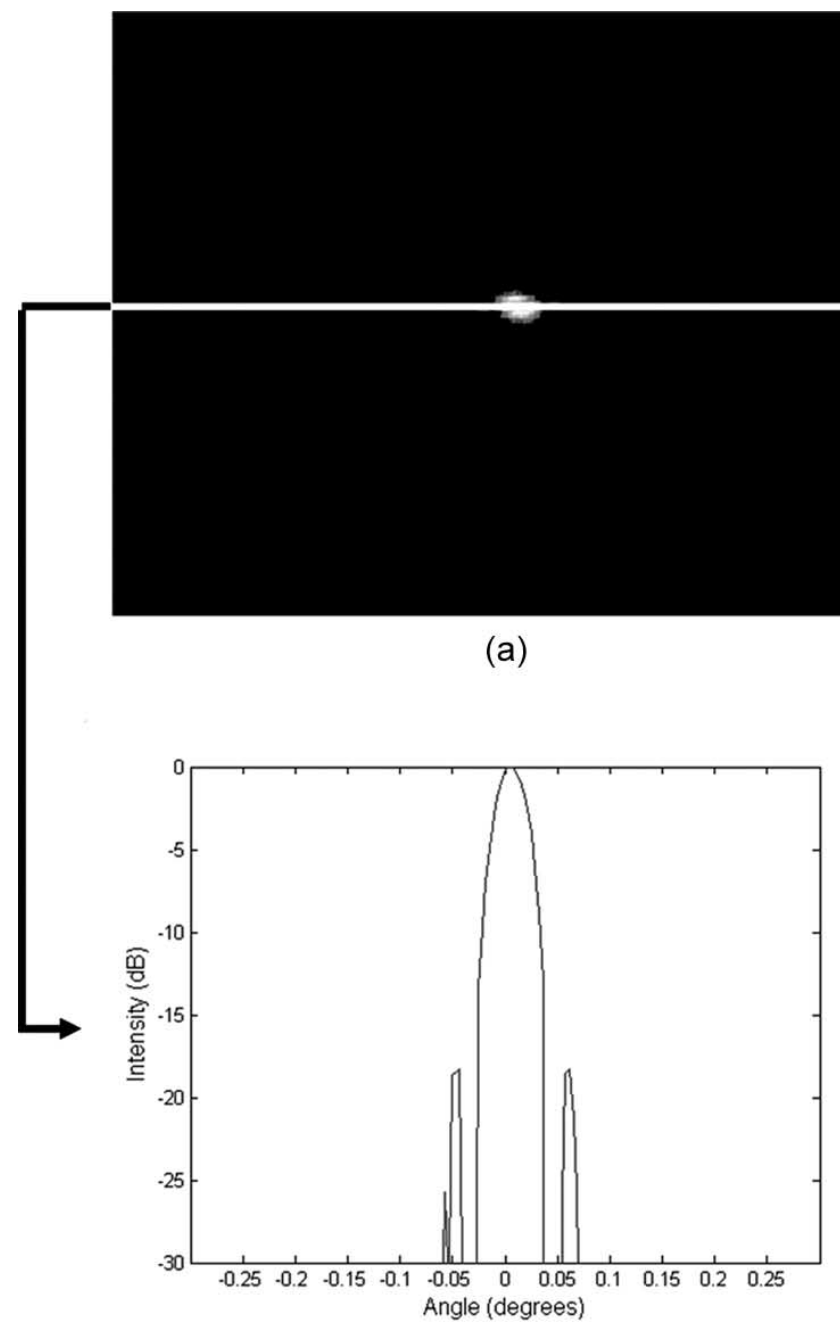

(c)

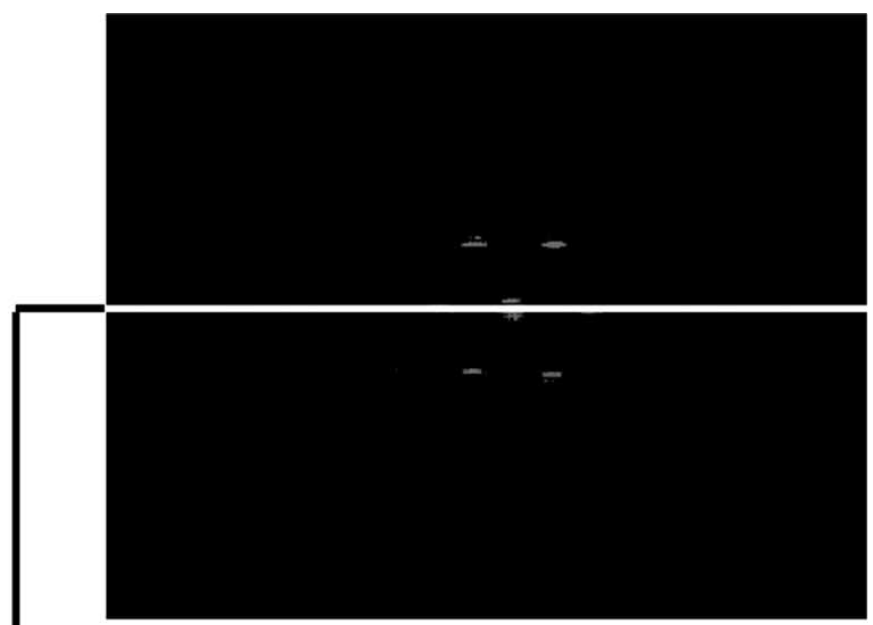

(b)

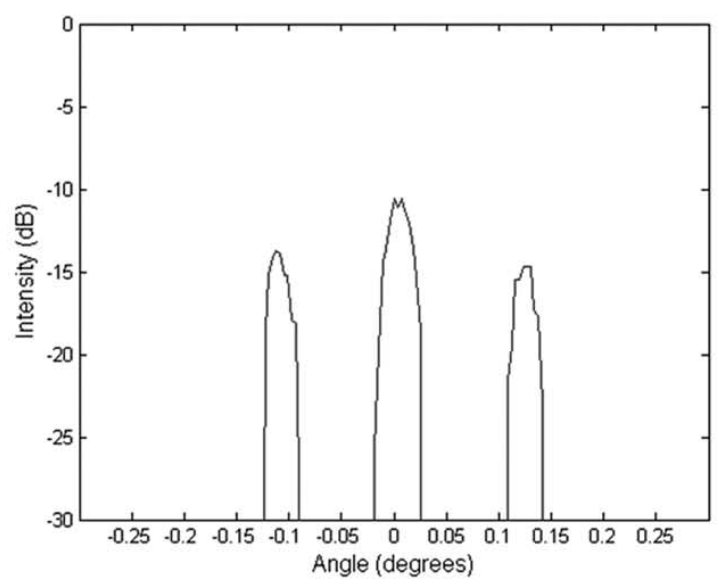

(d)

Fig. 13. Far-field diffraction pattern from the retromodulator, tilted $31.7^{\circ}$ towards the deformable mirror, with an applied voltage of (a) 0 and (b) 79 V. Photos and plots both use a logarithmic intensity scale.

$(0.55 \mu \mathrm{m})$ that could be achieved by our samples. According to Fig. 4, from our calculations, a correctly fabricated device will achieve $17-\mathrm{dB}$ extinction with $0.8-\mu \mathrm{m}$ deformation.

\section{B. Angular Dependence}

The retroreflecting modulator is used in an FSO communication system to automate the alignment at one side of the link; therefore, retroreflecting modulators must function at a wide range of incident angles. Accordingly, we tested the MEMS deformable-mirror retromodulator at a range of angles. Our simulations, shown in Fig. 4, predicted a decrease in contrast with an increase in $\alpha$, the angle between the incident light and the deformable-mirror-surface normal. We note that this angle $\alpha$ is not the same as the normal of the corner cube since each mirror is tilted $54.7^{\circ}$ relative to the corner-cube face. We measured the extinction ratios in relation to this angle and show these results in Fig. 15, where both $\alpha$ and the tilt of the corner cube $\beta$ are displayed. These results agree with our calculations of angular dependence and show a 2:1 extinction from $23^{\circ}$ to $58^{\circ}$ for $10-\mathrm{kHz}$ modulation. Thus, this particular device demonstrated a functional angular range of at least $35^{\circ}$. These extinction ratios are in strong agreement with the simulations presented in Fig. 4. Based on these calculations, we expect that a correctly fabricated fully deformable device can achieve higher extinctions and, therefore, can operate with a much greater angular range.

\section{Wavelength Dependence}

Fig. 16 shows the extinction ratio as a function of wavelength. It reveals constant extinction from the 1520-to-1570$\mathrm{nm}$ wavelength, which is consistent with our calculations. This indicates that the MEMS deformable retromodulator will reliably operate at a large range of wavelengths without a measurable impact on contrast.

\section{CONCLUSION}

We have presented a novel MEMS retromodulator for free-space optical communications and especially for remotetelemetry applications. This retromodulator combines excellent 


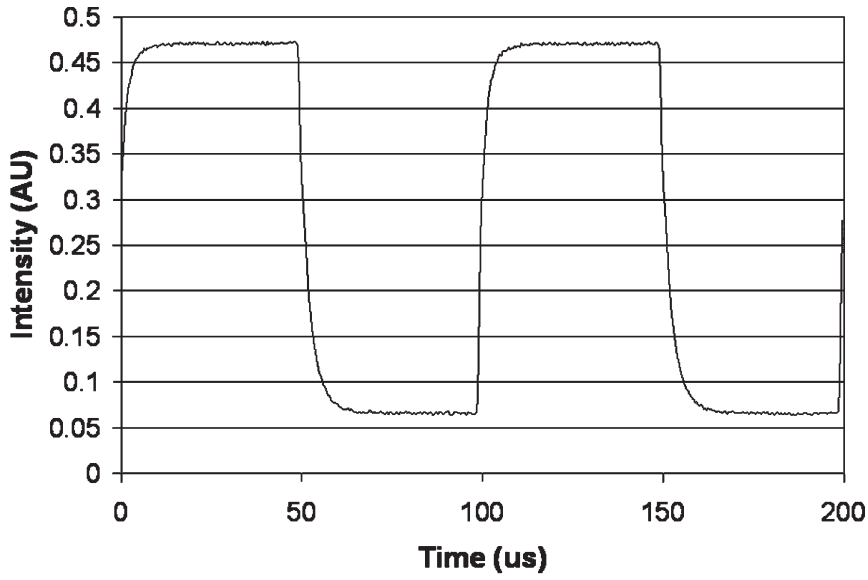

(a)

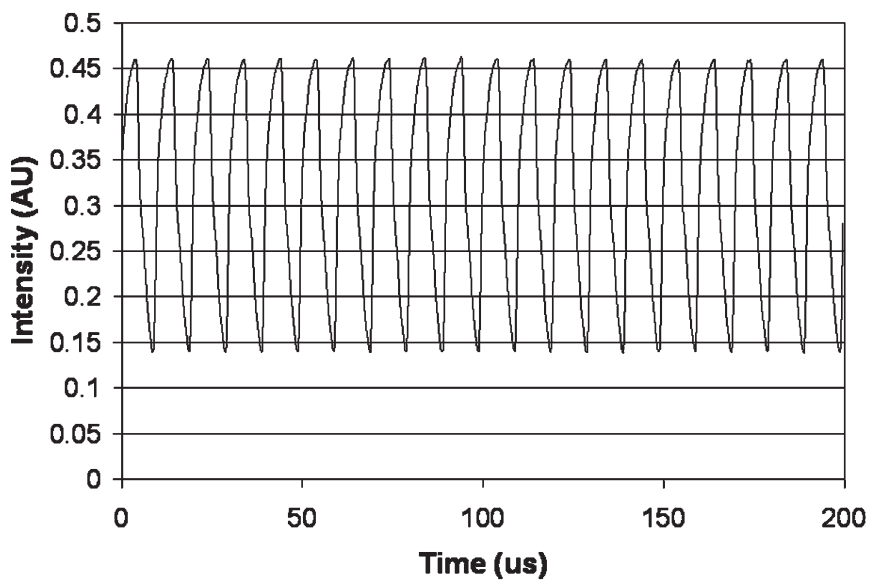

(b)

Fig. 14. Received (a) $10-\mathrm{kHz}$ and (b) 100-kHz signal from the retromodulator situated 6.6-m away.

wavelength, and angle and temperature insensitivity, while maintaining scalability for large apertures and longer link distances. Our theoretical analysis of the MEMS deformable mirror accurately describes the device's diffraction performance in relation to the physical design of the mirror. The deformable mirror was fabricated and tested to verify the wavelength and angle insensitivity predicted by our calculations. We also demonstrated large thermal tolerance, observing consistent device performance from 20 to $100{ }^{\circ} \mathrm{C}$. Finally, we assembled a single-deformable-micromirror-array device into a prototype retromodulator that demonstrated up to 7:1 extinction and modulation speeds up to $100 \mathrm{kHz}$.

The deformable mirror was designed to achieve greater deformations but was limited by the initial device-fabrication run to a maximum mirror deformation of $0.55 \mu \mathrm{m}$, as opposed to the design value of $0.8 \mu \mathrm{m}$. Future device fabrications with better control on the composition of the silicon-nitride layer will enable better extinction ratios to extend the angular, temperature, and wavelength operating range. Increasing the strain of the deformable membrane can increase the achievable switching speeds to $1 \mathrm{MHz}$.

This particular device is the first example of a class of optical MEMS components based on spatially segmenting the physical optical aperture into an array of smaller subaperture devices

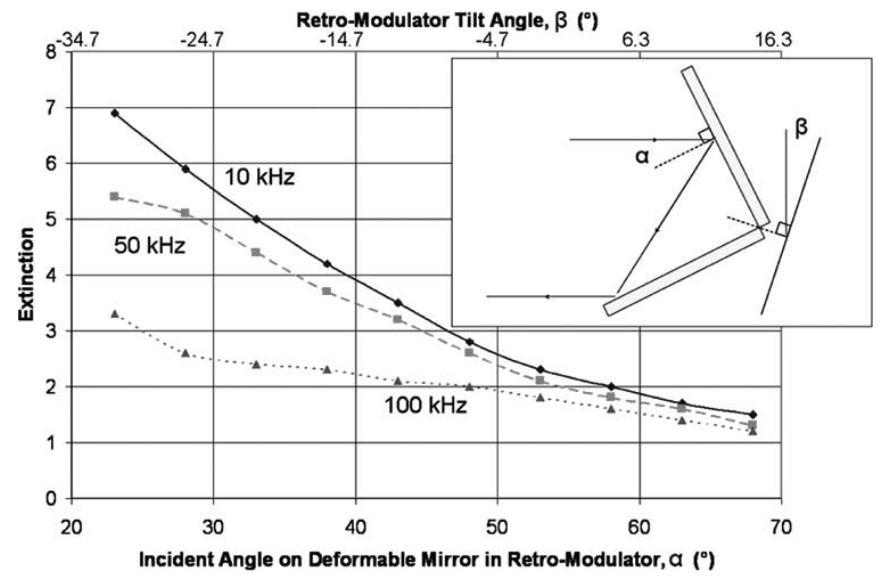

Fig. 15. Extinction ratio achieved as a function of retromodulator tilt angle.

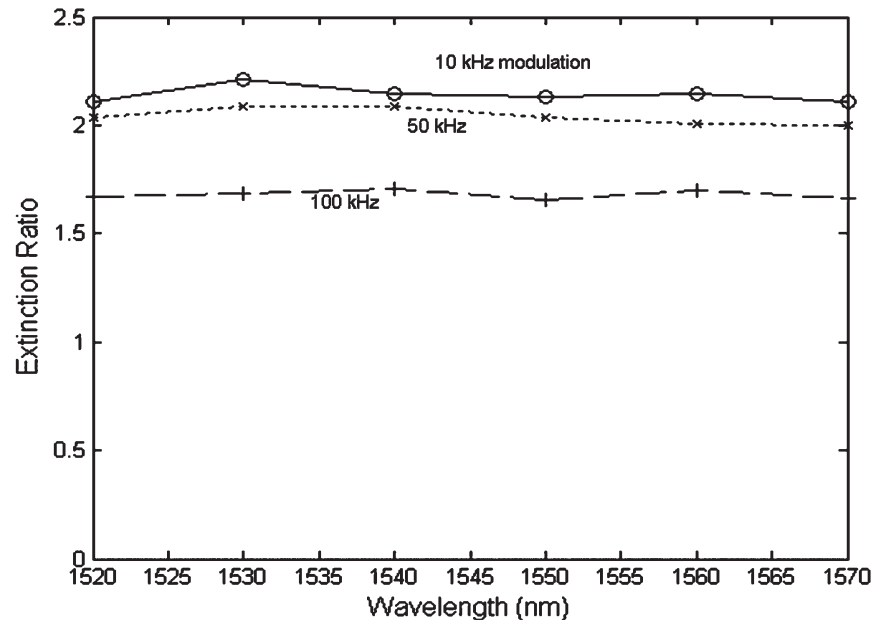

Fig. 16. Extinction as a function of wavelength, showing essentially wavelength-independent contrast.

that require a correspondingly small physical movement to control the optical response. This allows the device to scale to very-large physical apertures, limited only by the wafer size of device fabrication, and also enables a much faster mechanical response than would otherwise be possible. The particular design demonstrated here was an array of reflective micromirrors with variable focal length. This concept can be extended to other segmented structures, as for example an array of planar tilt mirrors, provided that one state of the MEMS device is uniformly flat to within a fraction of a wave across the full device array.

\section{ACKNOWLEDGMENT}

The authors acknowledge PROsystems, Inc., for fabricating the gold-mirror dihedral that they used as part of the prototype retromodulator.

\section{REFERENCES}

[1] L. Zhou, J. M. Kahn, and K. S. J. Pister, "Corner-cube retroreflectors based on structure-assisted assembly for free-space optical communication," J. Microelectromech. Syst., vol. 12, no. 3, pp. 233-242, Jun. 2003. 
[2] D. Pedersen and O. Solgaard, "Free space communication link using a grating light modulator," Sens. Actuators A, Phys., vol. 83, no. 1-3, pp. 6-10, May 2000.

[3] J. A. Walker, K. W. Goossen, and S. C. Arney, "Fabrication of a mechanical antireflection switch for fiber-to-the-home systems," J. Microelectromech. Syst., vol. 5, no. 1, pp. 45-51, Mar. 1996.

[4] J. E. Ford, J. A. Walker, D. S. Greywall, and K. W. Goossen, "Micromechanical fiber optic attenuator with $4 \mu$ s response," J. Lightw. Technol., vol. 16, no. 9, pp. 1663-1670, Sep. 1998.

[5] D. S. Greywall, P. A. Busch, and J. A. Walker, "Phenomenological model for gas-damping of micromechanical structures," Sens. Actuators A, Phys., vol. 72, no. 1, pp. 49-70, Jan. 1999.

[6] G. C. Gilbreath et al., "Progress in development of multiple-quantumwell retromodulators for free-space data links," Opt. Eng., vol. 42, no. 6, pp. 1611-1617, Jun. 2003

[7] C. Luo and K. W. Goossen, "Optical microelectromechanical system array for free-space retrocommunication," IEEE Photon. Technol. Lett., vol. 16, no. 9, pp. 2045-2047, Sep. 2004.

[8] J. Ford and J. Walker, "Technique for modulating optical signals in optical communications," U.S. Patent 5796880 , Aug. 18, 1998.

[9] S. Sakarya, G. Vdovin, and P. Sarro, "Micromachined SLM based on pixelated reflective membranes," in SPIE Proc., Denver, CO, 1999, vol. 3760, pp. 23-31.

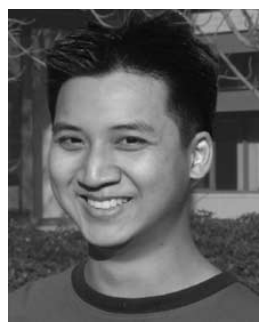

Trevor K. Chan received the B.Sc. degree in engineering physics at the University of Alberta, Edmonton, AB, Canada, in 2002, and the M.Sc. degree in electrical and computer engineering at the University of California, San Diego (UCSD), in 2004. He is currently working towards the Ph.D. degree in electrical and computer engineering.

He joined the Photonics Systems Integration Lab, UCSD, in 2003. His research interests include freespace optical communications, optical modulators, and wavelength-division-multiplexing technologies.

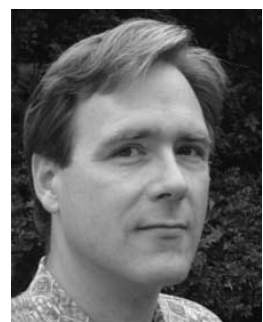

Joseph E. Ford received the Masters degrees in physics and optical engineering from the University of British Columbia,Vancouver, BC, Canada, and the University of Rochester, Rochester, NY, respectively, and the Ph.D. degree from the Electrical and Computer Engineering Department, University of California, San Diego (UCSD).

$\mathrm{He}$ was a member of Bell Labs' Advanced Photonics Research Department from 1994 to 2000, where he developed optoelectronics and microoptomechanics for parallel optical communications. From 2000 to 2002, he was with Optical Micro-Machines, where he ultimately became Chief Scientist. In 2002, he joined UCSD's Department of Electrical and Computer Engineering, where he leads the Photonic Systems Integration Laboratory. He is the coauthor of 33 refereed journal articles, 85 conference proceedings, and 44 U.S. patents on optical components and systems. 